\title{
Solvent-Free Green and Efficient One-Pot Synthesis of Dihydropyrano[3,2-c]chromene Derivatives
}

\author{
Shubha Jain, Deepika Rajguru, Balwant S. Keshwal, and Aman D. Acharya \\ School of Studies in Chemistry, Vikram University, Ujjain, Madhya Pradesh 456010, India \\ Correspondence should be addressed to Deepika Rajguru; deepika.rajguru23@gmail.com
}

Received 17 June 2013; Accepted 19 August 2013

Academic Editors: M. D’Auria, R. Pohl, D. Sémeril, and S. B. Wan

Copyright (c) 2013 Shubha Jain et al. This is an open access article distributed under the Creative Commons Attribution License, which permits unrestricted use, distribution, and reproduction in any medium, provided the original work is properly cited.

\begin{abstract}
A rapid, clean, and highly efficient method for synthesis of dihydropyrano[3,2-c]chromene derivatives by one-pot, three-component condensation of aromatic aldehydes, malononitrile, and 4-hydroxycoumarin using DABCO as catalyst in solvent-free neat conditions is described. The present method has the advantages of mild reaction conditions, short reaction times, easy isolation of products, and excellent yields.
\end{abstract}

\section{Introduction}

Multicomponent reactions (MCRs) are very important in organic synthesis due to the formation of carbon-carbon and carbon-hetero atom bonds in one pot [1-3]. Simple procedures, high bond forming efficiency, time and energy saving, and low expenditures are among the advantages of these reactions [4]. Over the past several years, chemists have been aware of the environmental implications of their chemistry. Nowadays, they are trying to develop new synthetic methods, reaction conditions, and uses of chemicals that reduce the risks to humans and the environment. Organic solvents are high on the list of hazardous chemicals because they are used in large amounts and are usually volatile liquids. Therefore, in recent years, solventless organic reactions have attracted great interest. They have many advantages such as high efficiency and selectivity, operational simplicity, low costs, mild reaction conditions, and reduced pollution [5-7]. Pyrano[3,2-c]chromenes are a class of important heterocycles with a wide range of biological properties [8] such as spasmolytic, diuretic, anticoagulant, anticancer, and antianaphylactic activity [9]. Moreover, they have been used as cognitive enhancers, for the treatment of neurodegenerative diseases, including Alzheimer's disease, Parkinson's disease, Huntington's disease, amyotrophic lateral sclerosis, AIDS associated dementia, and Down's syndrome as well as for the treatment of schizophrenia and myoclonus
[10]. In addition, aminochromene derivatives exhibit a wide spectrum of biological activities including antihypertensive and anti-ischemic behavior [11-13].

Several methods have been reported for the synthesis of pyrano[3,2-c]chromene derivatives. 2-Amino-4-aryl-5-oxo$4 H, 5 H$-pyrano[3,2-c]chromene-3-carbonitriles have previously been prepared from aromatic aldehydes, malononitrile, and 4-hydroxycoumarin in the presence of organic bases like piperidine or pyridine in an organic solvent, that is, ethanol and pyridine [14]. They have also been prepared in the presence of diammonium hydrogen phosphate (DAHP), (S)-proline [15], $\mathrm{K}_{2} \mathrm{CO}_{3}$ under microwave irradiation [16], TBAB [17], $\mathrm{MgO}$ [18], $\mathrm{H}_{6} \mathrm{P}_{2} \mathrm{~W}_{18} \mathrm{O}_{62} \cdot 18 \mathrm{H}_{2} \mathrm{O}$ [19], Hexamethylene tetramine [20], TMGT [21], $N, N, N^{\prime}, N^{\prime}$-tetrabromo benzene-1,3-disulfonamide (TBBDA) and poly $\left(N, N^{\prime}\right.$ dibromo- $N$-ethyl-benzene-1,3-disulfonamide) (PBBS) [22], 3-hydroxypropanaminium acetate (HPAA) [23], 2-hydroxyethylammonium formate [24], [bmim] Br [25], potassium phthalimide- $\mathrm{N}$-oxyl [26], and $\mathrm{CuO}$ nanoparticles [27]. However, some of these methods suffer from the serious limitations such as long reaction times, multistep reactions, complex synthetic pathways, and lower product yields. Therefore, the development of milder, faster, and more ecofriendly methods, accompanied with higher yields is needed.

In recent years, 1,4-diazabicyclo[2.2.2] octane (DABCO) has received considerable attention as an inexpensive, ecofriendly, high reactive, easy to handle, and nontoxic base 
catalyst for various organic transformations, affording the corresponding products in excellent yields with high selectivity $[28,29]$. The reactions are environmentally friendly and the catalyst can be recycled in some cases. In continuation of our interest to further enlarge the application of DABCO as a catalyst [30], here we wish to report one-pot synthesis of pyrano[3,2-c]chromene derivatives by the reaction of aromatic aldehydes, malononitrile, and 4-hydroxycoumarin catalyzed by DABCO in solventless conditions.

\section{Results and Discussion}

When aromatic aldehyde $\mathbf{1}$, malononitrile 2, and 4-hydroxy coumarin 3 were condensed in the presence of DABCO in solvent-free neat conditions at $100^{\circ} \mathrm{C}$; 2-Amino-4-aryl3-cyano-5-oxo-4H,5H-pyrano[3,2-c] chromene derivatives 4 were obtained in good to high yields (Scheme 1).

In our initial study, the reaction of benzaldehyde, malononitrile, and 4-hydroxycoumarin was used as a model reaction to optimize the reaction conditions. First the reaction was conducted in various solvents using DABCO as a catalyst under refluxing conditions and also under solventfree conditions. As can be seen from Table 1, the best results were obtained in neat. The effect of temperature in solventless conditions was studied by carrying out the reaction at 60,80 , 100 , and $120^{\circ} \mathrm{C}$. The results from Table 1 (entry 6 ) showed that $100^{\circ} \mathrm{C}$ would be the best temperature for all reactions.

Under the optimized reaction conditions, a series of dihydropyrano[3,2-c] chromene derivatives $\mathbf{4}(\mathbf{a}-\mathbf{j})$ were synthesized. The results are summarized in Table 2 . In all cases, aromatic aldehydes substituted with either electron-donating or electron-withdrawing groups underwent the reaction smoothly and gave the expected products in good to high yields under the same reaction conditions. Moreover, heteroaromatic aldehydes could also be successfully converted to the corresponding heteroaryl substituted pyrano[3,2c]chromenes in excellent yields.

\section{Conclusion}

In summary, a new clean and efficient protocol for the synthesis of pyrano[3,2-c]chromene derivatives using DABCO under solvent-free conditions was described. The use of $\mathrm{DABCO}$ as a green, nontoxic, nonexplosive, inexpensive, nonvolatile, easy to handle, and thermally stable catalyst with simple experimental and isolation procedure makes it an attractive method for the preparation of these compounds.

\section{Experimental}

4.1. General. All chemicals were purchased from Merck and Sigma-Aldrich as "synthesis grade" and used without further purification. Melting points were determined in open glass capillaries and are uncorrected. ${ }^{1} \mathrm{H}$ NMR spectra were obtained at $400 \mathrm{MHz}$ with a Bruker (AVANCE) spectrometer using DMSO- $\mathrm{d}_{6}$ as solvent and TMS as an internal standard. Elemental analysis was performed using Carlo Erba-1108 analyzer.
4.2. General Procedure for the Synthesis of 2-Amino-4-aryl-5oxo-4H,5H-pyrano[3,2-c]chromene-3-carbonitriles. Aromatic aldehyde 1 ( $1 \mathrm{mmoL})$, malononitrile $2(1.2 \mathrm{mmoL})$, 4-hydroxycoumarin 3 ( $1 \mathrm{mmoL})$, and DABCO (5 moL\%) were mixed thoroughly and heated in a water bath at $100^{\circ} \mathrm{C}$ for appropriate time. After completion of the reaction (monitored by TLC), the mixture was cooled to room temperature. The solid product was washed with hot water dried and recrystallized from ethanol to give the pure product.

All the compounds were characterized by spectroscopic and physical data which were found to be identical to those described in the literature.

4.2.1. 2-Amino-5-oxo-4-phenyl-4,5-dihydropyrano[3,2-c]chromene-3-carbonitrile (4a). White Solid, Yield: 94\%; m.p.256$257^{\circ} \mathrm{C}\left(256-258^{\circ} \mathrm{C}\right)$ [15]; Anal. Calcd. for $\mathrm{C}_{19} \mathrm{H}_{12} \mathrm{~N}_{2} \mathrm{O}_{3}$ : C, 72.15; H, 3.82; N, 8.86\%. Found: C, 72.28; H, 3.61; N, 8.65\%; ${ }^{1} \mathrm{H}-\mathrm{NMR}\left(400 \mathrm{MHz}, \mathrm{DMSO}-\mathrm{d}_{6}, \delta / \mathrm{ppm}\right): 4.16(1 \mathrm{H}, s, \mathrm{CH})$, $6.34(2 \mathrm{H}, s$, amino group), 7.35-7.44 (5H, $m, \mathrm{ArH}), 7.53-7.58$ (2 H, $m, \mathrm{ArH}), 7.65-7.71(2 \mathrm{H}, m, \mathrm{ArH}) ;{ }^{13} \mathrm{C}-\mathrm{NMR}(400 \mathrm{MHz}$, DMSO- $\left.\mathrm{d}_{6}, \delta / \mathrm{ppm}\right): 53.27,103.62,113.38,115.83,116.01,121.32$, $123.14,123.53,124.85,125.08,127.12,128.37,141.35,152.78$, $154.84,158.19,159.65$.

4.2.2. 2-Amino-4-(4-nitrophenyl)-5-oxo-4,5-dihydropyrano[3, 2-c]chromene-3-carbonitrile (4b). Pale Yellow Solid, Yield: $96 \%$; m.p. $259-261^{\circ} \mathrm{C}\left(258-260^{\circ} \mathrm{C}\right)$ [15]; Anal. Calcd. for $\mathrm{C}_{19} \mathrm{H}_{11} \mathrm{~N}_{3} \mathrm{O}_{5}$ : C, 63.16; H, 3.07; N, 11.63\%. Found: C, 63.29; $\mathrm{H}, 3.19$; N, 11.95\%; ${ }^{1} \mathrm{H}-\mathrm{NMR}$ (400 MHz, DMSO-d 6 , $\left.\delta / \mathrm{ppm}\right)$ : $4.76(1 \mathrm{H}, s, \mathrm{CH}), 6.43(2 \mathrm{H}, s$, amino group), $7.20-7.22(2 \mathrm{H}$, $\left.d d, J_{a}=3.9 \mathrm{~Hz}, J_{b}=1.0 \mathrm{~Hz}, \operatorname{ArH}\right), 7.27(1 \mathrm{H}, s, \mathrm{ArH}), 7.42$ $(1 \mathrm{H}, d, J=3.6 \mathrm{~Hz}, \mathrm{ArH}), 7.63-7.65\left(1 \mathrm{H}, d d, J_{a}=4.2 \mathrm{~Hz}, J_{b}\right.$ $=0.9 \mathrm{~Hz}, \operatorname{ArH}), 7.69(1 \mathrm{H}, d, J=3.7 \mathrm{~Hz}, \operatorname{ArH}), 7.76(2 \mathrm{H}, d$, $J=1.2 \mathrm{~Hz}, \mathrm{ArH}) ;{ }^{13} \mathrm{C}-\mathrm{NMR}\left(400 \mathrm{MHz}, \mathrm{DMSO}_{6}, \delta / \mathrm{ppm}\right)$ : $57.34,105.83,112.56,115.91,116.73,119.26,122.06,123.12,125.49$, $126.64,129.03,138.23,148.39,152.84,153.17,158.35,159.46$.

4.2.3. 2-Amino-4-(3-nitrophenyl)-5-oxo-4,5-dihydropyrano[3, 2-c]chromene-3-carbonitrile (4c). White Solid, Yield: 93\%; m.p. 261-263 ${ }^{\circ} \mathrm{C}\left(262-264^{\circ} \mathrm{C}\right)$ [15]; Anal. Calcd. for $\mathrm{C}_{19} \mathrm{H}_{11} \mathrm{~N}_{3} \mathrm{O}_{5}$ : C, 63.16; H, 3.07; N, 11.63\%. Found: C, 63.28; $\mathrm{H}, 3.17$; N, 11.96\%; ${ }^{1} \mathrm{H}-\mathrm{NMR}\left(400 \mathrm{MHz}, \mathrm{DMSO}-\mathrm{d}_{6}, \delta / \mathrm{ppm}\right)$ : $4.26(1 \mathrm{H}, s, \mathrm{CH}), 6.32(2 \mathrm{H}, s$, amino group), 7.15-7.24 $(3 \mathrm{H}$, $m, \operatorname{ArH}), 7.46(2 \mathrm{H}, d, J=7.6 \mathrm{~Hz}, \operatorname{ArH}), 7.90(1 \mathrm{H}, d, J=$ $2.0 \mathrm{~Hz}, \mathrm{ArH}), 8.33(1 \mathrm{H}, d, J=7.6 \mathrm{~Hz}, \mathrm{ArH}), 8.87(1 \mathrm{H}, s, \mathrm{ArH})$; ${ }^{13} \mathrm{C}-\mathrm{NMR}\left(400 \mathrm{MHz}, \mathrm{DMSO}_{6}, \delta / \mathrm{ppm}\right): 55.37,107.58$, $113.35,115.19,116.24,119.73,122.52,123.65,125.54,128.73$, $129.46,135.48,148.36,152.11,153.72,158.02,159.78$.

4.2.4. 2-Amino-4-(4-chlorophenyl)-5-oxo-4,5-dihydropyrano [3,2-c]chromene-3-carbonitrile (4d). White Solid, Yield: 92\%; m.p. $264-267^{\circ} \mathrm{C}\left(263-265^{\circ} \mathrm{C}\right)$ [15]; Anal. Calcd. for $\mathrm{C}_{19} \mathrm{H}_{11} \mathrm{ClN}_{2} \mathrm{O}_{3}$ : C, 65.06; H, 3.16; N, 7.99\%. Found: C, 65.35; $\mathrm{H}, 3.26$; N, 7.73\%; ${ }^{1} \mathrm{H}-\mathrm{NMR}\left(400 \mathrm{MHz}, \mathrm{DMSO}-\mathrm{d}_{6}, \delta / \mathrm{ppm}\right)$ : $4.72(1 \mathrm{H}, s, \mathrm{CH}), 6.74(2 \mathrm{H}, s$, amino group), $7.22(1 \mathrm{H}, t, J=$ $4.6 \mathrm{~Hz}, \mathrm{ArH}), 7.70-7.73(3 \mathrm{H}, m, \mathrm{ArH}), 7.84(2 \mathrm{H}, d, J=8.6 \mathrm{~Hz}$, $\mathrm{ArH}), 8.36(2 \mathrm{H}, d, J=8.6 \mathrm{~Hz}, \mathrm{ArH}) ;{ }^{13} \mathrm{C}-\mathrm{NMR}(400 \mathrm{MHz}$, 


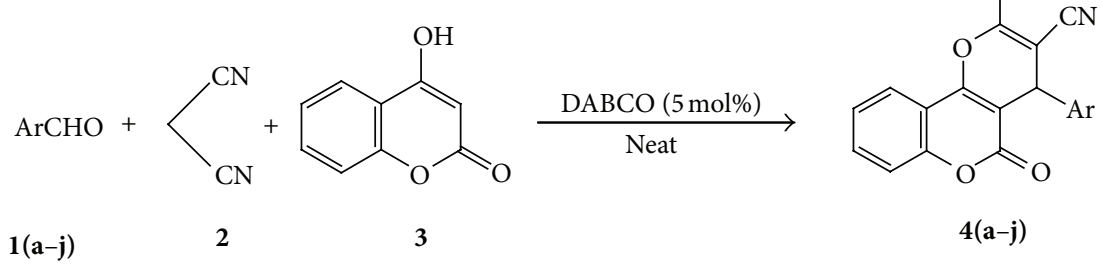

SCHEME 1

TABLE 1: DABCO catalyzed synthesis of $\mathbf{4 a}$ in different reaction conditions.

\begin{tabular}{lcccc}
\hline Entry & Solvent & $\mathrm{T} /{ }^{\circ} \mathrm{C}$ & Time/h & Yield $^{\mathrm{a}} \%$ \\
\hline 1 & $\mathrm{EtOH}$ & Reflux & 2 & 85 \\
2 & $\mathrm{CH}_{2} \mathrm{Cl}_{2}$ & Reflux & 6 & 54 \\
3 & $\mathrm{CH}_{3} \mathrm{CN}$ & Reflux & 4 & 73 \\
4 & $\mathrm{THF}$ & Reflux & 4 & 62 \\
5 & $\mathrm{H}_{2} \mathrm{O}$ & Reflux & 1.5 & 87 \\
6 & - & 100 & 0.5 & 94 \\
7 & - & 60 & 2 & 65 \\
8 & - & 80 & 1 & 76 \\
9 & - & 120 & 0.5 & 92 \\
$10^{\mathrm{b}}$ & - & 100 & 2 & 65 \\
$11^{\mathrm{c}}$ & - & 100 & 0.5 & 95 \\
\hline
\end{tabular}

${ }^{\mathrm{a}}$ Isolated yield.

${ }^{\mathrm{b}} 1 \mathrm{~mol} \%$ of catalyst was used.

${ }^{c} 10 \mathrm{~mol} \%$ of catalyst was used.

DMSO-d $\left.{ }_{6}, \delta / p p m\right): 54.72,106.43,112.78,115.62,116.58,119.32$, $121.09,123.45,124.64,125.92,128.53,134.32,138.10,152.18$, $152.97,158.49,159.56$.

4.2.5. 2-Amino-4-(4-bromophenyl)-5-oxo-4,5-dihydropyrano [3,2-c]chromene-3-carbonitrile (4e). White Solid, Yield: $91 \%$; m.p. 247-250 $\mathrm{C}\left(249-251^{\circ} \mathrm{C}\right)$ [15]; Anal. Calcd. for $\mathrm{C}_{19} \mathrm{H}_{11} \mathrm{BrN}_{2} \mathrm{O}_{3}$ : C, 57.74; H, 2.81; N, 7.09\%. Found: C, 57.53; $\mathrm{H}, 2.96$; N, 7.17\%; ${ }^{1} \mathrm{H}-\mathrm{NMR}$ (400 MHz, DMSO-d 6 , $\left.\delta / \mathrm{ppm}\right)$ : $4.36(1 \mathrm{H}, s, \mathrm{CH}), 6.60(2 \mathrm{H}, s$, amino group), $7.44(2 \mathrm{H}, d, J$ $=3.5 \mathrm{~Hz}, \mathrm{ArH}), 7.77(1 \mathrm{H}, d, J=1.7 \mathrm{~Hz}, \mathrm{ArH}), 7.82-7.84(3 \mathrm{H}$, $m, \mathrm{ArH}), 8.36-8.38\left(2 \mathrm{H}, d d, J_{a}=4.9 \mathrm{~Hz}, J_{b}=1.9 \mathrm{~Hz}, \mathrm{ArH}\right)$; ${ }^{13} \mathrm{C}-\mathrm{NMR}\left(400 \mathrm{MHz}, \mathrm{DMSO}-\mathrm{d}_{6}, \delta / \mathrm{ppm}\right): 55.43,105.57$, $112.58,115.23,116.62,119.82,121.08,123.42,124.78,126.56$, $127.58,136.45,139.34,152.49,152.84,157.37,159.89$.

4.2.6. 2-Amino-4-(4-methoxyphenyl)-5-oxo-4,5-dihydropyrano[3,2-c]chromene-3-carbonitrile (4f). White Solid, Yield: 89\%; m.p. 241-244 ${ }^{\circ} \mathrm{C}\left(240-242^{\circ} \mathrm{C}\right)$ [15]; Anal. Calcd. for $\mathrm{C}_{20} \mathrm{H}_{14} \mathrm{~N}_{2} \mathrm{O}_{4}$ : C, 69.36; H, 4.07; N, 8.09\%. Found: C, 68.92; $\mathrm{H}, 4.20$; N, 8.19\%; ${ }^{1} \mathrm{H}-\mathrm{NMR}\left(400 \mathrm{MHz}, \mathrm{DMSO}-\mathrm{d}_{6}, \delta / \mathrm{ppm}\right)$ : $3.76\left(3 \mathrm{H}, s, \mathrm{OCH}_{3}\right), 5.13(1 \mathrm{H}, s, \mathrm{CH}), 6.36(2 \mathrm{H}, s$, amino group), 6.65-6.66 $(2 \mathrm{H}, q, J=1.7 \mathrm{~Hz}, \mathrm{ArH}), 7.41(2 \mathrm{H}, d, J=$ $3.3 \mathrm{~Hz}, \mathrm{ArH}), 7.57$ (2H, m, ArH), $7.75(2 \mathrm{H}, d, J=1.5 \mathrm{~Hz}, \mathrm{ArH})$; ${ }^{13} \mathrm{C}-\mathrm{NMR}\left(400 \mathrm{MHz}, \mathrm{DMSO}-\mathrm{d}_{6}, \delta / \mathrm{ppm}\right): 52.95,57.66,103.90$, $112.96,116.26,117.39,119.28,122.49,123.72,124.32,125.26$, $126.51,132.42,138.10,152.01,152.95,158.24,159.53$.
4.2.7. 2-Amino-5-oxo-4-p-tolyl-4,5-dihydropyrano[3,2-c]chromene-3-carbonitrile (4g). White Solid, Yield: 87\%; m.p. 252$254^{\circ} \mathrm{C}\left(250-252^{\circ} \mathrm{C}\right)$ [15]; Anal. Calcd. for $\mathrm{C}_{20} \mathrm{H}_{14} \mathrm{~N}_{2} \mathrm{O}_{3}$ : C, 72.72; H, 4.27; N, 8.48\%. Found: C, 72.46; H, 4.19; N, 8.58\%; ${ }^{1} \mathrm{H}-\mathrm{NMR}\left(400 \mathrm{MHz}, \mathrm{DMSO}-\mathrm{d}_{6}, \delta / \mathrm{ppm}\right): 2.10\left(3 \mathrm{H}, s, \mathrm{CH}_{3}\right)$, $4.58(1 \mathrm{H}, s, \mathrm{CH}), 6.73(2 \mathrm{H}, s$, amino group), $7.22-7.24(4 \mathrm{H}$, $m, \operatorname{ArH}), 7.70-7.71\left(2 \mathrm{H}, d d, J_{a}=2.7 \mathrm{~Hz}, J_{b}=1.0 \mathrm{~Hz}, \mathrm{ArH}\right)$, $7.74-7.76\left(2 \mathrm{H}, d d, J_{a}=4.2 \mathrm{~Hz}, J_{b}=1.0 \mathrm{~Hz}, \mathrm{ArH}\right) ;{ }^{3} \mathrm{C}-\mathrm{NMR}$ $\left(400 \mathrm{MHz}, \mathrm{DMSO}_{6}, \delta / \mathrm{ppm}\right): 13.95,30.57,55.59,101.64$, $111.65,112.92,116.24,118.88,122.44,124.20,132.47,146.91$, $150.86,151.93,152.08,153.90,158.74,159.35$.

4.2.8. 2-Amino-4-(2,4-dichlorophenyl)-5-oxo-4,5-dihydropyrano[3,2-c]chromene-3-carbonitrile (4h). White Solid, Yield: 90\%; m.p. $256-258^{\circ} \mathrm{C}\left(257-259^{\circ} \mathrm{C}\right)$ [15]; Anal. Calcd. for $\mathrm{C}_{19} \mathrm{H}_{10} \mathrm{Cl}_{2} \mathrm{~N}_{2} \mathrm{O}_{3}$ : C, 59.24; H, 2.62; N, 7.27\%. Found: C, 59.51; $\mathrm{H}, 2.49$; N, 7.48\%; ${ }^{1} \mathrm{H}-\mathrm{NMR}\left(400 \mathrm{MHz}, \mathrm{DMSO}-\mathrm{d}_{6}, \delta / \mathrm{ppm}\right)$ : $4.24(1 \mathrm{H}, s, \mathrm{CH}), 6.31(2 \mathrm{H}, s$, amino group), $6.66(1 \mathrm{H}, d$, $J=3.1 \mathrm{~Hz}, \mathrm{ArH}), 6.71(1 \mathrm{H}, t, J=1.4 \mathrm{~Hz}, \mathrm{ArH}), 7.30(1 \mathrm{H}$, $d, J=3.2 \mathrm{~Hz}, \mathrm{ArH}), 7.59$ (2H, $m, \mathrm{ArH}), 7.85(1 \mathrm{H}, s, \mathrm{ArH})$, $7.95(1 \mathrm{H}, d, J=0.9 \mathrm{~Hz}, \mathrm{ArH}) ;{ }^{13} \mathrm{C}-\mathrm{NMR}(400 \mathrm{MHz}, \mathrm{DMSO}-$ $\left.\mathrm{d}_{6}, \delta / \mathrm{ppm}\right): 28.38,57.99,104.05,113.36,115.97,116.19,119.36$, $120.50,122.40,123.38,124.23,125.34,127.15,132.26,135.03$, $151.95,152.77,158.15,159.53$.

4.2.9. 2-Amino-4-(furan-2-yl)-5-oxo-4,5-dihydropyrano[3,2c] chromene-3-carbonitrile (4i). Brown Solid, Yield: 97\%; m.p. 251-254 ${ }^{\circ} \mathrm{C}\left(252-253^{\circ} \mathrm{C}\right)$ [16]; Anal. Calcd. for $\mathrm{C}_{17} \mathrm{H}_{10} \mathrm{~N}_{2} \mathrm{O}_{4}$ : C, 66.67; H, 3.29; N, 9.15\%. Found: C, 66.95; H, 3.07; N, 9.27\%; ${ }^{1} \mathrm{H}-\mathrm{NMR}\left(400 \mathrm{MHz}, \mathrm{DMSO}-\mathrm{d}_{6}, \delta / \mathrm{ppm}\right): 3.92(1 \mathrm{H}, s, \mathrm{CH})$, $6.30\left(2 \mathrm{H}, s\right.$, amino group), $6.79-6.81\left(1 \mathrm{H}, d d, J_{a}=4.9 \mathrm{~Hz}\right.$, $J_{b}=1.8 \mathrm{~Hz}$, furan ring), 7.08-7.12 $(3 \mathrm{H}, m$, furan ring+ArH), $7.38-7.41\left(1 \mathrm{H}, d d, J_{a}=3.4 \mathrm{~Hz}, J_{b}=1.6 \mathrm{~Hz}, \operatorname{ArH}\right), 7.75(1 \mathrm{H}, d$, $J=2.5 \mathrm{~Hz}, \mathrm{ArH}), 7.86(1 \mathrm{H}, d, J=4.7 \mathrm{~Hz}, \mathrm{ArH}) ;{ }^{13} \mathrm{C}-\mathrm{NMR}$ (400 MHz, DMSO-d 6 , $\delta / \mathrm{ppm}): 30.49,55.87,101.64,106.16$, $106.98,111.55,112.90,116.17,118.86,122.44,124.11,150.92,151.67$, $152.05,153.94,158.71,159.50$.

4.2.10. 2-Amino-5-oxo-4-(thiophen-2-yl)-4,5-dihydropyrano[3,2-c]chromene-3-carbonitrile (4j). White Solid, Yield: 96\%; m.p. $226-230^{\circ} \mathrm{C}\left(228^{\circ} \mathrm{C}\right)$ [20]; Anal. Calcd. for $\mathrm{C}_{17} \mathrm{H}_{10} \mathrm{~N}_{2} \mathrm{O}_{3} \mathrm{~S}$ : C, 63.34; H, 3.13; N, 8.69; S, 9.95\%. Found: C, 63.53; H, 3.24; N, 8.37; S, 9.68\%; ${ }^{1} \mathrm{H}-\mathrm{NMR}$ (400 MHz, DMSO-d $\left.\mathrm{d}_{6}, \delta / \mathrm{ppm}\right): 4.94$ $(1 \mathrm{H}, s, \mathrm{CH}), 6.54(2 \mathrm{H}, s$, amino group $), 6.98(1 \mathrm{H}, m$, thiophene ring), $7.20-7.22\left(2 \mathrm{H}, d d, J_{a}=3.8 \mathrm{~Hz}, J_{b}=1.3 \mathrm{~Hz}\right.$, thiophene ring), $7.65-7.67\left(2 \mathrm{H}, d d, J_{a}=4.2 \mathrm{~Hz}, J_{b}=1.0 \mathrm{~Hz}, \mathrm{ArH}\right)$, 7.69-7.70 $\left(2 \mathrm{H}, d d, J_{a}=2.6 \mathrm{~Hz}, J_{b}=1.1 \mathrm{~Hz}, \mathrm{ArH}\right) ;{ }^{13} \mathrm{C}-\mathrm{NMR}$ 
TABLE 2: Synthesis of 2-Amino-4-aryl-3-cyano-5-oxo-4H,5H-pyrano[3,2-c]chromenes in solvent-free neat conditions using DABCO $(5 \mathrm{~mol} \%)$ as catalyst.

\begin{tabular}{|c|c|c|c|c|c|}
\hline Entry & $\mathrm{Ar}$ & Product & Time/min & Yield $/ \%$ & m.p. $/{ }^{\circ} \mathrm{C}$ Found (reported) \\
\hline 1 & $\mathrm{C}_{6} \mathrm{H}_{5}$ & $4 a$ & 30 & 94 & $256-257(256-258)[15]$ \\
\hline 2 & $4-\mathrm{O}_{2} \mathrm{~N} \mathrm{C}_{6} \mathrm{H}_{4}$ & $4 b$ & 30 & 96 & $259-261(258-260)[15]$ \\
\hline 3 & $3-\mathrm{O}_{2} \mathrm{~N} \mathrm{C}_{6} \mathrm{H}_{4}$ & $4 \mathrm{c}$ & 30 & 93 & $261-263(262-264)[15]$ \\
\hline 4 & $4-\mathrm{ClC}_{6} \mathrm{H}_{4}$ & $4 d$ & 30 & 92 & $264-267(263-265)[15]$ \\
\hline 5 & $4-\mathrm{BrC}_{6} \mathrm{H}_{4}$ & $4 e$ & 30 & 91 & $247-250(249-251)[15]$ \\
\hline 6 & $4-\mathrm{CH}_{3} \mathrm{OC}_{6} \mathrm{H}_{4}$ & $4 \mathrm{f}$ & 30 & 89 & $241-244(240-242)[15]$ \\
\hline 7 & $4-\mathrm{CH}_{3} \mathrm{C}_{6} \mathrm{H}_{4}$ & $4 \mathrm{~g}$ & 30 & 87 & $252-254(250-252)[15]$ \\
\hline 8 & $2,4-\mathrm{Cl}_{2} \mathrm{C}_{6} \mathrm{H}_{3}$ & $4 h$ & 30 & 90 & $256-258(257-259)[15]$ \\
\hline 9 & & $4 i$ & 30 & 97 & $251-254(252-253)[16]$ \\
\hline 10 & & $4 j$ & 30 & 96 & $226-230(228)[20]$ \\
\hline
\end{tabular}

(400 MHz, DMSO-d 6 , $\delta / \mathrm{ppm}): 31.94,57.84,103.97,116.22$, $118.94,122.51,124.27,124.58,124.67,127.72,132.52,138.41$, $140.90,152.95,153.84,158.40,159.49$.

\section{Acknowledgment}

The authors thank the director of SAIF, Punjab University, Chandigarh, for NMR spectral data.

\section{References}

[1] A. Domling, "Recent developments in isocyanide based multicomponent reactions in applied chemistry," Chemical Reviews, vol. 106, no. 1, pp. 17-89, 2006.

[2] I. Ugi, "Multicomponent reactions (MCR): Perspectives of multicomponent reactions and their libraries," Advanced Synthesis andCatalysis, vol. 339, no. 1, pp. 499-516, 1997.

[3] N. K. Terrett, M. Gardner, D. W. Gordon, R. J. Kobylecki, and J. Steele, "Combinatorial synthesis-the design of compound libraries and their application to drug discovery," Tetrahedron, vol. 51, no. 30, pp. 8135-8173, 1995.

[4] A. Domling and I. Ugi, "Multicomponent reactions with isocyanides," Angewandte Chemie, vol. 39, no. 18, pp. 3168-3210, 2000.

[5] K. Tanaka and F. Toda, "Solvent-free organic synthesis," Chemical Reviews, vol. 100, no. 3, pp. 1025-1074, 2000.

[6] F. Shirini, K. Marjani, and H. T. Nahzomi, "Silica triflate as an efficient catalyst for the solvent-free synthesis of 3,4dihydropyrimidin-2(1H)-ones," Arkivoc, vol. 2007, no. 1, pp. 51$57,2007$.

[7] G. Thirunarayanan and G. Vanangamudi, "Synthesis of some 4bromo-1-naphthyl chalcones using silica-sulfuric acid reagent under solvent free conditions," Arkivoc, vol. 2006, no. 12, pp. 58$64,2006$.
[8] G. R. Green, J. M. Evans, A. K. Vong, A. R. Katritzky, C. W. Rees, and E. F. Scriven, "Pyrans and their benzo derivatives synthesis," in Comprehensive Heterocyclic Chemistry II, vol. 5, p. 469, Pergamon Press, Oxford, UK, 1995.

[9] W. O. Foye, Principi Di Chemico Frmaceutica, Piccin, Padova, Italy, 1991.

[10] C. S. Konkoy, D. B. Fick, S. X. Cai, N. C. Lan, and J. F. W. Keana, "PCT international application WO0075123 (2000)," Chemical Abstracts, vol. 134, Article ID 29313a, 2001.

[11] A. Burgard, H. Lang, and U. Gerlach, "Asymmetric synthesis of 4-amino-3,4-dihydro-2,2-dimethyl-2H-1 benzopyrans," Tetrahedron, vol. 55, no. 24, pp. 7555-7562, 1999.

[12] J. M. Evans, C. S. Fake, T. C. Hamilton, R. H. Poyser, and G. A. Showell, "Synthesis and antihypertensive activity of 6,7-disubstituted trans-4-amino-3,4-dihydro-2,2-dimethyl2H-1-benzopyran-3-ols," Journal of Medicinal Chemistry, vol. 27, no. 9, pp. 1127-1131, 1984.

[13] J. M. Evans, C. S. Fake, T. C. Hamilton, R. H. Poyser, and E. A. Watts, "Synthesis and antihypertensive activity of substituted trans-4-amino-3,4-dihydro-2,2-dimethyl-2H-1benzopyran-3-ols," Journal of Medicinal Chemistry, vol. 26, no. 11, pp. 1582-1589, 1983.

[14] R. M. Shaker, "Synthesis and reactions of some new 4Hpyrano[3,2-c] benzopyran-5-one derivatives and their potential biological activities," Pharmazie, vol. 51, no. 3, pp. 148-151, 1996.

[15] A. S. Mohammadi and S. Balalaie, "Novel and efficient catalysts for the one-pot synthesis of 3,4-dihydropyrano[c]chromene derivatives in aqueous media," Tetrahedron Letters, vol. 48, pp. 3299-3303, 2007.

[16] M. Kidwai and S. Saxena, "Convenient preparation of pyrano benzopyranes in aqueous media," Synthetic Communications, vol. 36, no. 18, pp. 2737-2742, 2006.

[17] J. M. Khurana and S. Kumar, "Tetrabutylammonium bromide (TBAB): a neutral and efficient catalyst for the synthesis of biscoumarin and 3,4-dihydropyrano[ $c]$ chromene derivatives in 
water and solvent-free conditions," Tetrahedron Letters, vol. 50, no. 28, pp. 4125-4127, 2009.

[18] M. Seifi and H. Sheibani, "High surface area $\mathrm{MgO}$ as a highly effective heterogeneous base catalyst for threecomponent synthesis of tetrahydrobenzopyran and 3,4dihydropyrano $[c]$ chromene derivatives in aqueous media," Catalysis Letters, vol. 126, no. 3-4, pp. 275-279, 2008.

[19] M. M. Heravi, B. A. Jani, F. Derikvand, F. F. Bamoharram, and H. A. Oskooie, "Three component, one-pot synthesis of dihydropyrano[3,2-c]chromene derivatives in the presence of $\mathrm{H}_{6} \mathrm{P}_{2} \mathrm{~W}_{18} \mathrm{O}_{62} \cdot 18 \mathrm{H}_{2} \mathrm{O}$ as a green and recyclable catalyst," Catalysis Communications, vol. 10, no. 3, pp. 272-275, 2008.

[20] H. J. Wang, J. Lu, and Z. H. Zhang, "Highly efficient three-component, one-pot synthesis of dihydropyrano[3,2c]chromene derivatives," Monatshefte für Chemie, vol. 141, no. 10, pp. 1107-1112, 2010.

[21] A. Shaabani, S. Samadi, Z. Badri, and A. Rahmati, "Ionic liquid promoted efficient and rapid one-pot synthesis of pyran annulated heterocyclic systems," Catalysis Letters, vol. 104, no. 1-2, pp. 39-43, 2005.

[22] G. V. Ramin, T. S. Zahra, and K. N. Rahman, “One-pot synthesis of $4 \mathrm{H}$-Chromene and Dihydropyrano[3,2-c]chromene derivatives in hydroalcoholic media," Journal of the Brazilian Chemical Society, vol. 22, no. 5, pp. 905-909, 2011.

[23] H. R. Shaterian and A. R. Oveisi, "A simple Green approach to the synthesis of 2-amino-5-oxo-4,5-dihydropyrano[3,2c]chromene-3-carbonitrile derivatives catalyzed by 3hydroxypropanaminium acetate (HPAA) as a new ionic liquid," Journal of the Iranian Chemical Society, vol. 8, no. 2, pp. 545-552, 2011.

[24] H. R. Shaterian, M. Arman, and F. Rigi, "Domino Knoevenagel condensation, Michael addition, and cyclization using ionic liquid, 2-hydroxyethylammonium formate, as a recoverable catalyst," Journal of Molecular Liquids, vol. 158, no. 2, pp. 145150, 2011.

[25] N. Tavakoli-Hoseini, M. M. Heravi, F. F. Bamoharram, and A. Davoodnia, "Green, catalyst-free protocol for synthesis of dihydropyrano $[3,2-c]$ chromenes in ionic liquid [bmim]Br as an efficient promoting medium," Asian Journal of Chemistry, vol. 23, no. 8, pp. 3599-3601, 2011.

[26] M. G. Dekamin, M. Eslami, and A. Maleki, "Potassium phthalimide- $N$-oxyl: a novel, efficient, and simple organocatalyst for the one-pot three-component synthesis of various 2amino- $4 H$-chromene derivatives in water," Tetrahedron, vol. 69, no. 3, pp. 1074-1085, 2013.

[27] M. Hossein and K. M. Maryam, "CuO nanoparticles: an efficient and recyclable nanocatalyst for the rapid and green synthesis of 3,4-dihydropyrano[c]chromenes," Chinese Chemical Letters, vol. 22, no. 12, pp. 1419-1422, 2011.

[28] H. Yang, R. Tian, and Y. Li, "Organic reactions catalyzed by 1 , 4-diazabicyclo [2.2.2] octane (DABCO)," Frontiers of Chemistry in China, vol. 3, no. 3, pp. 279-287, 2008.

[29] B. Baghernejad, "1,4-Diazabicyclo[2.2.2] octane (DABCO) as a useful catalyst in organic synthesis," European Journal of Chemistry, vol. 1, no. 1, pp. 54-60, 2010.

[30] P. Paliwal, S. R. Jetti, A. Bhatewara et al., "DABCO catalyzed synthesis of Xanthene derivatives in aqueous media," ISRN Organic Chemistry, vol. 2013, Article ID 526173, 6 pages, 2013. 

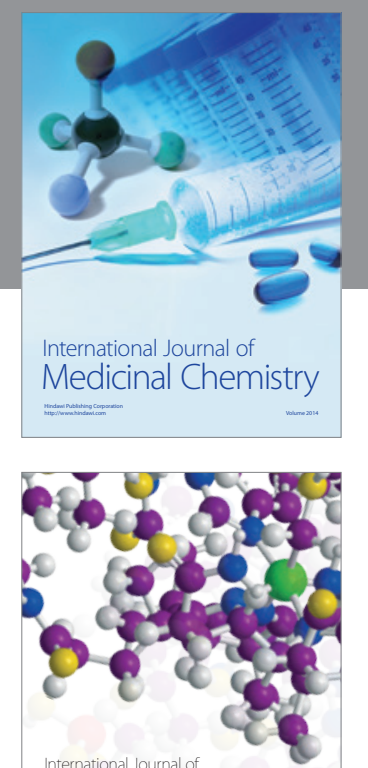

\section{Carbohydrate} Chemistry

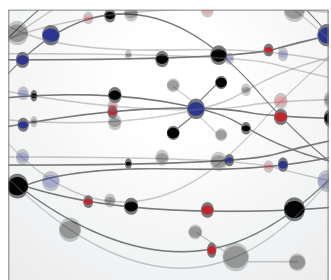

The Scientific World Journal
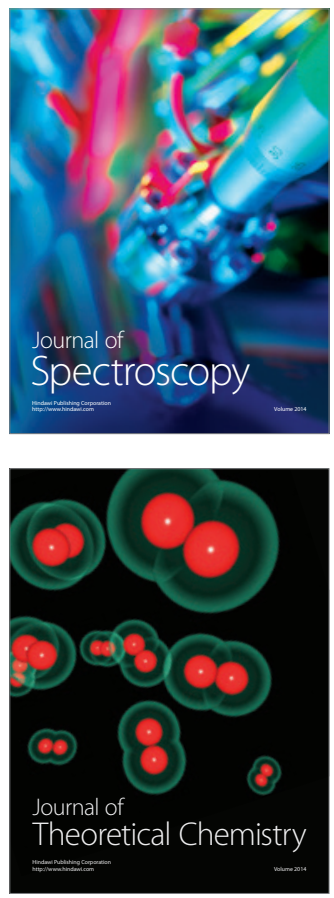
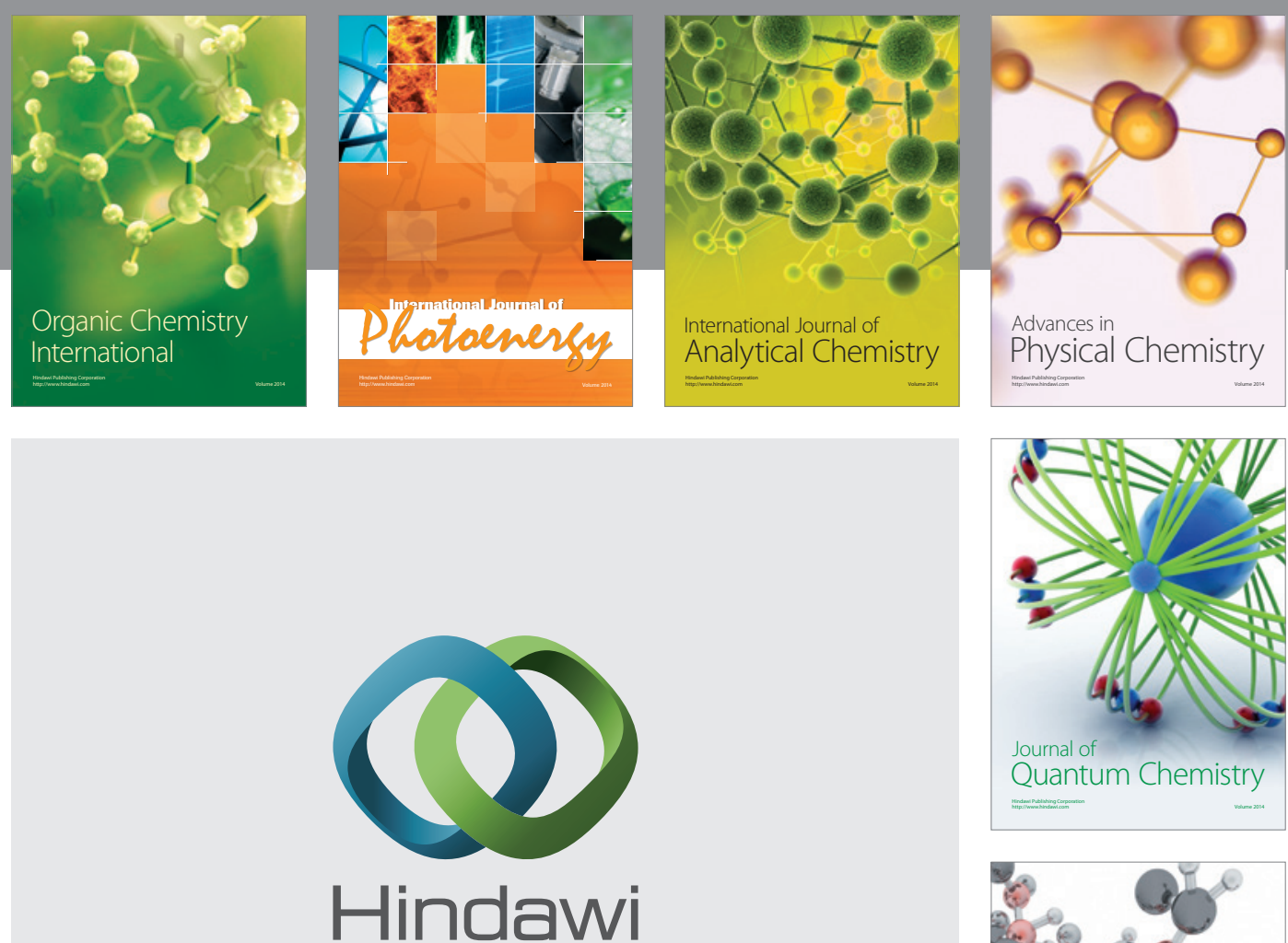

Submit your manuscripts at

http://www.hindawi.com

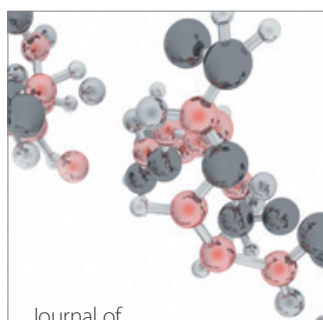

Analytical Methods

in Chemistry

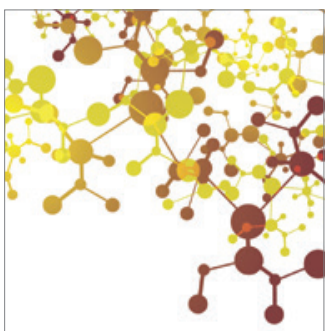

Journal of

Applied Chemistry

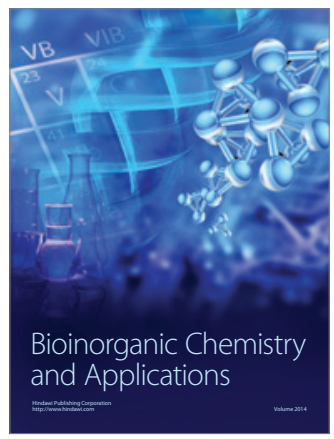

Inorganic Chemistry
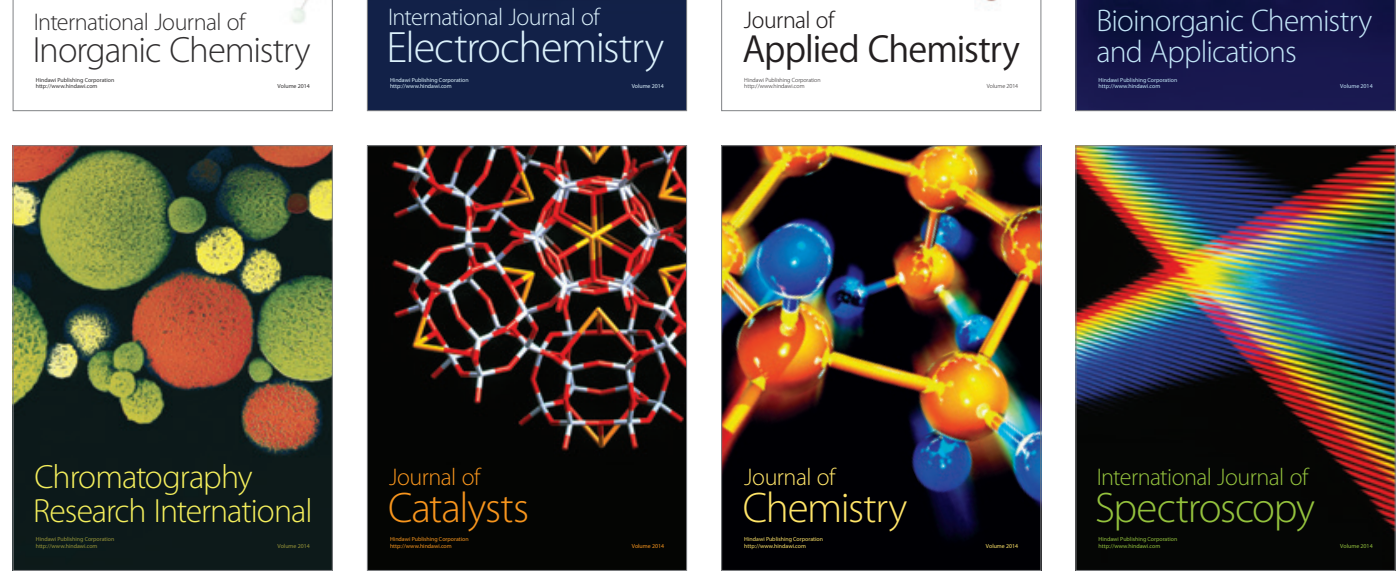